\title{
Genetic analysis of mitochondrial protein misfolding in Drosophila melanogaster
}

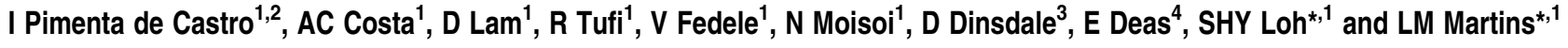

Protein misfolding has a key role in several neurological disorders including Parkinson's disease. Although a clear mechanism for such proteinopathic diseases is well established when aggregated proteins accumulate in the cytosol, cell nucleus, endoplasmic reticulum and extracellular space, little is known about the role of protein aggregation in the mitochondria. Here we show that mutations in both human and fly PINK1 result in higher levels of misfolded components of respiratory complexes and increase in markers of the mitochondrial unfolded protein response. Through the development of a genetic model of mitochondrial protein misfolding employing Drosophila melanogaster, we show that the in vivo accumulation of an unfolded protein in mitochondria results in the activation of AMP-activated protein kinase-dependent autophagy and phenocopies of pink1 and parkin mutants. Parkin expression acts to clear mitochondria with enhanced levels of misfolded proteins by promoting their autophagic degradation in vivo, and refractory to Sigma $P($ ref(2)P), the Drosophila orthologue of mammalian p62, is a critical downstream effector of this quality control pathway. We show that in flies, a pathway involving pink1, parkin and ref(2)P has a role in the maintenance of a viable pool of cellular mitochondria by promoting organellar quality control.

Cell Death and Differentiation (2012) 19, 1308-1316; doi:10.1038/cdd.2012.5; published online 3 February 2012

Aberrant protein folding is linked to several neurological disorders including Parkinson's disease (PD). Such proteinopathic diseases are often linked to the formation of intracellular aggregates of toxic proteins in the cytosol, cell nucleus, endoplasmic reticulum and extracellular space (reviewed in Rubinsztein ${ }^{1}$ ). Additionally, the deterioration of functional mitochondria is thought to have important consequences for the function and survival of neurons, and has therefore been proposed to have a causative role in neurological disorders such as PD (reviewed in de Castro et $a l^{2}$ ). In spite of the evidence linking both protein aggregation and mitochondrial dysfunction to PD, surprisingly little is known whether mitochondrial protein aggregates can also have a significant role in neurodegenerative diseases.

Recently, the PD-associated kinase PINK1 was identified as an important modulator of the mitochondrial serine protease HtrA2 activity. ${ }^{3}$ Deletion of $H t r A 2$ in mice results in the accumulation of unfolded proteins in the mitochondria, indicating that protein aggregation in this organelle may contribute to the advancement of neurodegenerative diseases. ${ }^{4}$ Additionally, the ubiquitin ligase Parkin has been shown to act in organellar quality control, to promote the autophagy of damaged mitochondria ${ }^{5}$ through a PINK1 recruitment mechanism. ${ }^{6}$ Thus, PINK1 seems to act as an upstream modulator of both molecular and organellar quality control pathways via HtrA2 and Parkin, respectively.

Through the analysis of post-mortem brains from PD patients carrying PINK1 mutations that affect the phosphorylation status of the serine protease $\mathrm{HtrA} 2$, we detected the presence of enhanced levels of misfolded components of mitochondrial respiratory complexes as well as an increase in the levels of the mitochondrial HSP-60, a marker of the activation of the mitochondrial unfolded protein response $\left(\mathrm{UPR}^{\mathrm{mt}}\right.$ ) in the nematode Caenorhabditis elegans (reviewed in Broadle and $\mathrm{Hartl}^{7}$ ). These results indicate that the accumulation of protein aggregates in the mitochondria might be detrimental to mitochondrial function and led us to develop an in vivo model for the selective accumulation of misfolded proteins in this organelle.

To dissect the in vivo consequences of mitochondrial protein aggregation, we make use of the fruit fly, Drosophila melanogaster. Here, we show that Drosophila mutants in pink1 and parkin display higher levels of misfolded components of mitochondrial respiratory complexes and an increase in the levels of the mitochondrial HSP-60 (reviewed in Broadle and $\mathrm{Hartl}^{7}$ ). Using a novel genetic model of mitochondrial protein misfolding, we show that the in vivo accumulation of an unfolded protein causes generalised mitochondrial dysfunction and accompanied by engagement of autophagy in an AMP-activated protein kinase (AMPK)-dependent manner.

${ }^{1}$ Cell Death Regulation, MRC Toxicology Unit, Lancaster Road, Leicester LE1 9HN, UK; ${ }^{3}$ Imaging and Pathology Laboratories, MRC Toxicology Unit, Lancaster Road, Leicester LE1 9HN, UK; ${ }^{2}$ IPATIMUP and Faculty of Pharmacy, University of Porto, Porto, Portugal and ${ }^{4}$ Department of Molecular Neuroscience, Institute of Neurology, Queen Square, London WC1N 3BG, UK

${ }^{*}$ Corresponding authors: LM Martins or SHY Loh, Cell Death Regulation Laboratory, MRC Toxicology Unit, Hodgkin Building, Lancaster Road, Leicester LE1 9HN, UK. Tel: + 44116252 5533; Fax: +44 116252 5616; E-mail: martins.Imiguel@ gmail.com (LMM) or Tel: + 44116223 1501; Fax: + 441162525616 ;

E-mail: SHYL1@LE.AC.UK (SHYL)

Keywords: unfolded proteins; mitochondria; Drosophila; autophagy

Abbreviations: ACC, acetyl-COA carboxylase; ALS, amyotrophic lateral sclerosis; AMPK, $5^{\prime}$ AMP-activated protein kinase; ANOVA, analysis of variance; ATP, adenosine triphosphate; IPD, idiopathic Parkinson's disease; OTC, ornithine transcarbamilase; PD, Parkinson's disease; ROS, reactive oxygen species; UBL, ubiquitin-like domain; $U_{P R}{ }^{\mathrm{ER}}$, endoplasmic reticulum unfolded-protein response; UPR ${ }^{\mathrm{mt}}$, mitochondrial unfolded protein response; UPS, ubiquitin-proteasome system Received 04.8.11; revised 19.12.11; accepted 09.1.12; Edited by H Steller; published online 03.2.12 
This genetic model of mitochondrial protein misfolding phenocopies pink1 and parkin mutants, suggesting that a compromise on mitochondrial protein quality control might be related to mitochondrial dysfunction caused by the loss of Pink1 or Parkin. Our study also suggests that Parkin expression acts to clear defective mitochondria with high levels of unfolded proteins by promoting their autophagic degradation in vivo, and refractory to Sigma $P(\operatorname{ref}(2) P)$, the Drosophila orthologue of mammalian p62, is a critical downstream effector of this quality control pathway.

\section{Results}

A novel Drosophila model of mitochondrial protein misfolding. To determine whether PINK1 dysfunction in humans is linked to protein conformational stress, we analysed the levels of misfolded mitochondrial respiratory components in brain samples from deceased PD patients, using an approach designed to investigate mitochondrial protein misfolding in HtrA2 mutant mice. ${ }^{4}$ In this analysis, we included PD patients harbouring heterozygous PINK1 mutations as well as idiopathic disease subjects (IPD) with no identified mutations in this gene. This revealed that patients carrying either the $\mathrm{Y} 431 \mathrm{H}$ or the $\mathrm{C} 575 \mathrm{R}$ mutations have significant levels of unfolded mitochondrial respiratory complexes (Figure 1a). Interestingly, levels of human HSP-60 positively correlate with the degree of misfolded respiratory complexes in these patients, suggesting that in humans protein conformational stress in the mitochondria is potentially linked to the activation of the UPR ${ }^{\mathrm{mt}}$. Notably, we have previously reported that patients carrying either the $\mathrm{Y} 431 \mathrm{H}$ or the $\mathrm{C575R}$ mutations have decreased levels of phospho-HtrA2, and are therefore likely to have defective activation of the mitochondrial serine protease $\mathrm{HtrA} 2$, a protein implicated in mitochondrial stress response. ${ }^{3}$

To determine whether Drosophila mutants of either pink1 or parkin mimicked the accumulation of unfolded proteins in mitochondria observed in PD patients, we analysed the levels of insoluble respiratory complexes in these flies. We observed enrichment of several respiratory complex subunits in both mutants (Figures $1 b$ and $c$ ), suggesting that these proteins are misfolded in mitochondria with defective Pink1 or Parkin.

Comparatively, little is known about the consequences of protein misfolding in mitochondria in vivo as no genetic models are available to address this issue. To directly determine the significance of protein misfolding in this organelle, we generated a genetic model of mitochondrial protein misfolding in Drosophila using a mutant form of the mitochondrial matrix protein, ornithine transcarbamilase (OTC) that accumulates in an unfolded state and that was first used to characterise the UPR ${ }^{\mathrm{mt}}$ in mammalian cells. ${ }^{8}$ Using the UAS/GAL4 ${ }^{9}$ system, we generated transgenic flies expressing the correctly folded version of this protein (OTC) as a control or the unfolded form (dOTC). After confirming that a

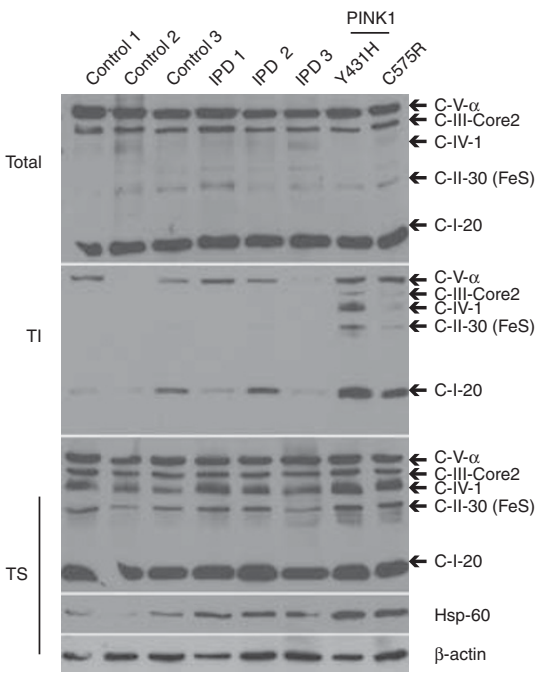

b
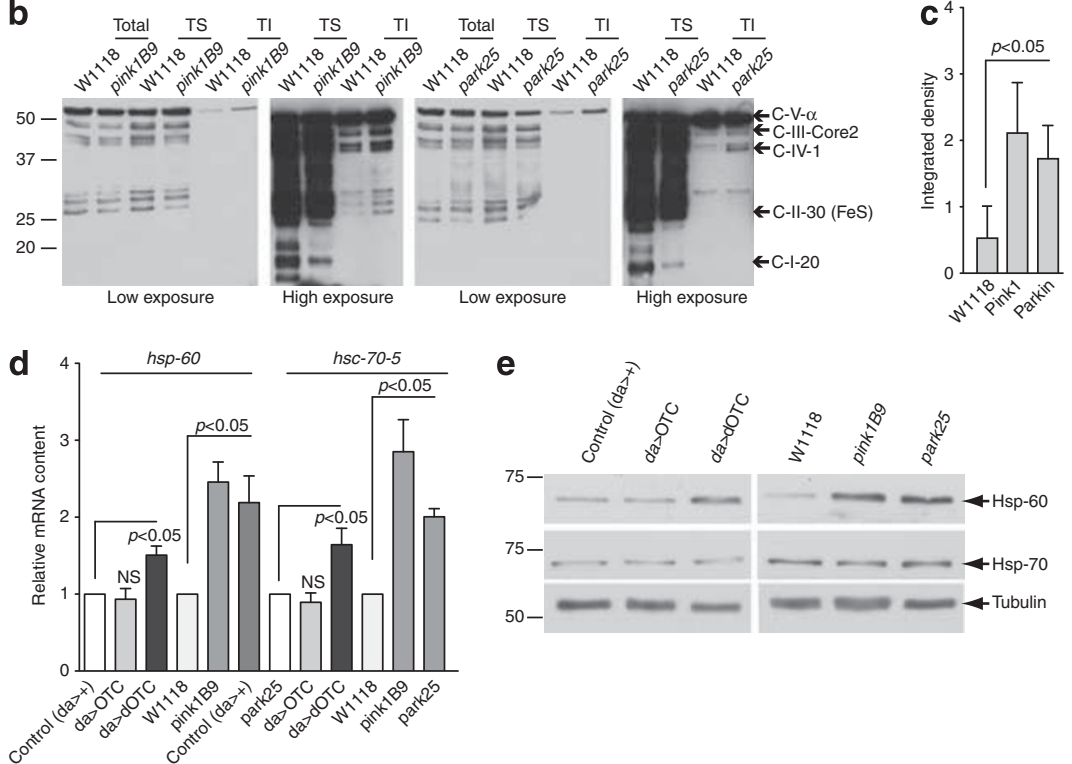

e
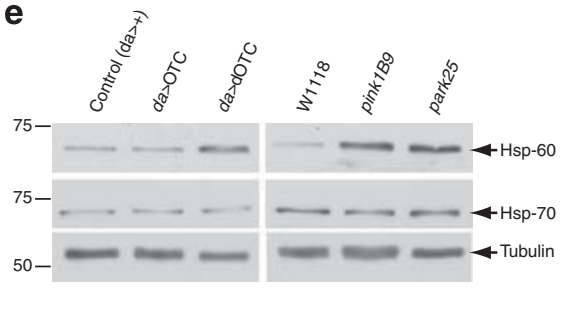

Figure 1 In vivo analysis of mitochondrial protein misfolding. (a) Analysis of respiratory complex solubility and HSP-60 levels in human brains. Human brain tissue (cortex) was analysed by western blotting. Normal control brains (control); idiopathic Parkinson's disease brains (IPD); and PD brains carrying mutant PINK1 were analysed. Protein fractions were prepared under gentle lysis conditions ${ }^{4}$ and compared with SDS-extracted proteins (Total). Total, Triton-soluble (TS) and Triton-insoluble (TI) fractions were subjected to western blot analysis using the indicated antibodies. (b) Analysis of respiratory complex solubility in Drosophila. Protein fractions of flies with the indicated genotypes were prepared under gentle lysis conditions ${ }^{4}$ and compared with SDS-extracted proteins (Total). Total, Triton-soluble (TS) and Triton-insoluble (TI) fractions were subjected to western blot analysis using the indicated antibodies. (c) Densitometry analysis was performed on three experiments representative of Figure $1 \mathrm{~b}$. Levels of unfolded respiratory complexes were normalised to SDS-extracted proteins (Total). Statistically significant values relative to control are indicated (one-way analysis of variance (ANOVA) with Dunnett's multiple comparison test). (d) Analysis of Drosophila hsp-60 and hsc-70-5 mRNA levels in dOTC transgenic flies, and Pink1 and parkin mutants. RNA was isolated from a pool of four flies and analysed by qRT-PCR. Data are shown as the mean \pm S.D. ( $n=3$ in each group). Statistically significant values relative to control are indicated (one-way ANOVA with Dunnett's multiple comparison test), and NS for $P>0.05$. (e) Analysis of the protein levels of mitochondrial HSP-60 and cytosolic HSP-70 upon expression of dOTC, and in Pink1 and parkin mutants. Lysates prepared from adult flies were subjected to western blot analysis with the indicated antibodies 
both proteins were being correctly targeted to mitochondria (Supplementary Figure S1A) and that dOTC accumulated in an insoluble form (Supplementary Figure S1B), we proceed with the characterisation of this fly model.

Protein conformational stress in mitochondria has been shown to lead to activation of the UPR ${ }^{\mathrm{mt}}$ characterised by the induction of the mitochondrial chaperones $h s p-60$ and $h s p-6$ in the nematode Caenorhabditis elegans. ${ }^{10}$ We observed a significant upregulation of the mRNA levels for $h s p-60$ as well as $h s c-70-5$, the Drosophila orthologue of $C$. elegans $h s p-6$, in flies where dOTC was expressed ubiquitously using the da-GAL4 driver; as well as in pink1 and parkin mutant animals (Figure 1d). This was accompanied by a significant induction of the protein levels of mitochondrial HSP-60 in dOTC, pink1 and parkin mutants but not for the cytosolic HSP-70 (Figure 1e), supporting the view that accumulation of unfolded proteins in the mitochondrial compartment and loss of pink 1 or parkin results in the selective upregulation of target genes encoding mitochondrial stress proteins in flies. We have also detected an upregulation of HSP-60 in flies treated with Paraquat, a pesticide linked to PD by epidemiological studies, known to result in high levels of reactive oxygen species (ROS) and consistent with its damaging effect on mitochondrial proteins (Supplementary Figure S1C).

We then crossed dOTC flies with genetic reporters of both the cytosolic ubiquitin-proteasome system (UPS) and the endoplasmic reticulum unfolded-protein response (UPR ${ }^{\mathrm{ER}}$ ). These experiments failed to reveal any impairment of the UPS or activation of the UPR ${ }^{\mathrm{ER}}$ in flies expressing dOTC (Supplementary Figure S2), further confirming the specificity of UPR ${ }^{\mathrm{mt}}$ activation by protein conformational stress in Drosophila.

We next assayed mitochondrial activity in flies expressing dOTC and controls by measuring state III respiration, and detected a significant decrease in oxygen consumption
(Figure 2a, and Supplementary Figures S1D, E) and in the adenosine triphosphate (ATP) levels (Figure $2 b$ ), suggesting defective mitochondrial function. These findings were accompanied by a loss of mitochondrial DNA and proteins (Figures 2c and d), likely towing to generalised mitochondrial loss. Strikingly, this mitochondrial loss was also observed in pink1 mutants. ${ }^{11}$ Taken together, these results suggest that excessive protein misfolding leads to compromised mitochondrial function and a loss of mitochondrial mass.

\section{Accumulation of misfolded proteins in mitochondria} phenocopies pink1 and parkin mutant flies. As we detected UPR ${ }^{\mathrm{mt}}$ activation in flies ubiquitously expressing dOTC and in parkin and pink1 mutant flies (Figure 1d), we decided to further investigate the phenotypic consequences of dOTC expression. We began to observe an abnormal (held up) wing posture in $10-15 \%$ of the flies expressing dOTC at 3 days after eclosion (Figures $3 a$ and b); therefore, we choose to analyse the indirect flight muscles of these animals. Expression of dOTC resulted in the formation of mitochondrial aggregates in the indirect flight muscles (Figures $3 \mathrm{c}$ and $\mathrm{d}$ ), and ultrastructural analysis showed a highly disorganised muscle structure, vacuolisation and fragmentation of mitochondrial cristae (Figures $3 e-h)$. Flies expressing dOTC also displayed an age-dependent impaired climbing ability, suggesting a locomotor deficit (Figure 3i) and reduced overall survival (Figure $3 \mathrm{j}$ ). We were able to detect a significant impairment in mitochondrial function in dOTCexpressing flies prior to any overt pathological changes, suggesting that a compromise in mitochondrial function lies at the apex of the detected pathologies. Interestingly, the phenotypes observed in this fly model of the UPR ${ }^{\mathrm{mt}}$ were similar to those of parkin and pink1 mutants, ${ }^{11-14}$ supporting
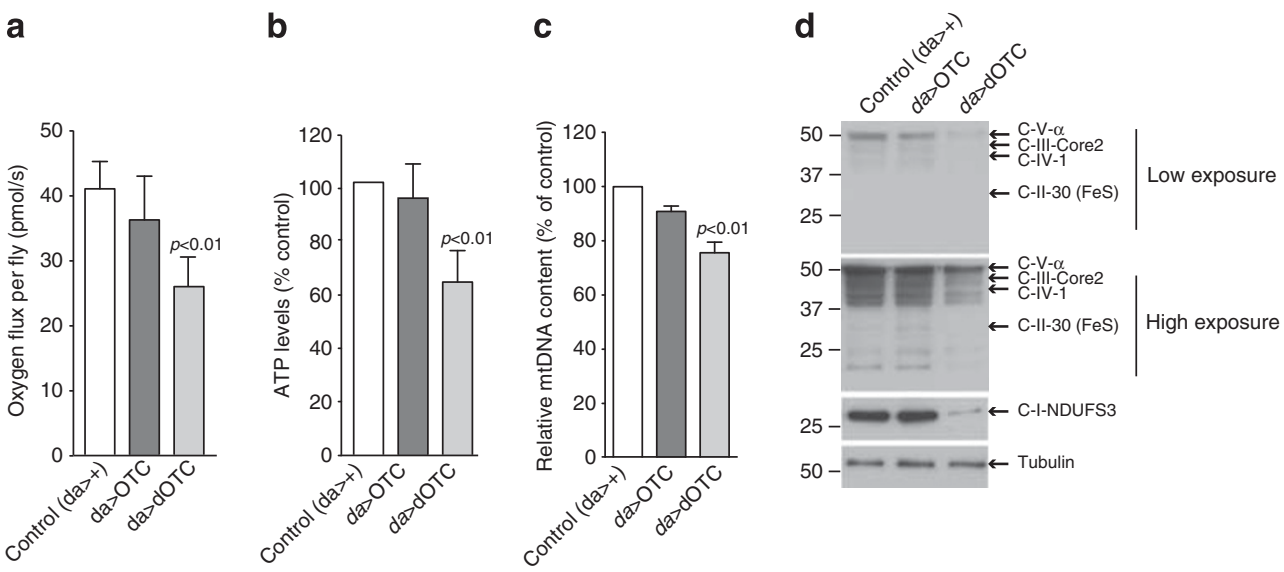

Figure 2 Accumulation of misfolded proteins in mitochondria results in mitochondrial impairment. (a) Defects in respiration in dOTC transgenic flies. Activity of the mitochondrial complex I was measured by high-resolution respirometry. Data are shown as the mean \pm S.D. ( $n=4$ in each group). Statistically significant values relative to control are indicated (one-way ANOVA with Dunnett's multiple comparison test). (b) dOTC expression resulted in generalised ATP loss. ATP levels were measured using a bioluminescence assay. Data are shown as the mean \pm S.D. ( $n=3$ in each group). Statistically significant values relative to control are indicated (one-way ANOVA with Dunnett's multiple comparison test). (c) dOTC expression led to a loss of mitochondrial DNA (mtDNA). The ratio of mtDNA to nuclear DNA was measured by real-time PCR on flies with the indicated genotypes (mean \pm S.D., $n=3$ in each group). Statistically significant values relative to control are indicated (one-way ANOVA with Dunnett's multiple comparison test). (d) Expression of dOTC led to a general decrease of mitochondrial respiratory complexes. Levels of soluble mitochondrial proteins in whole fly extracts were probed with a panel of antibodies raised against the nuclear-encoded subunit NDUFS 3 of complex I; complex II subunit $30 \mathrm{kDa}$; the nuclear-encoded subunit core 2 of complex III; the mitochondrial-encoded subunit I of complex IV and the ATP synthase subunit $\alpha$. Lysates prepared from adult flies were subjected to western blot analysis with the indicated antibodies 

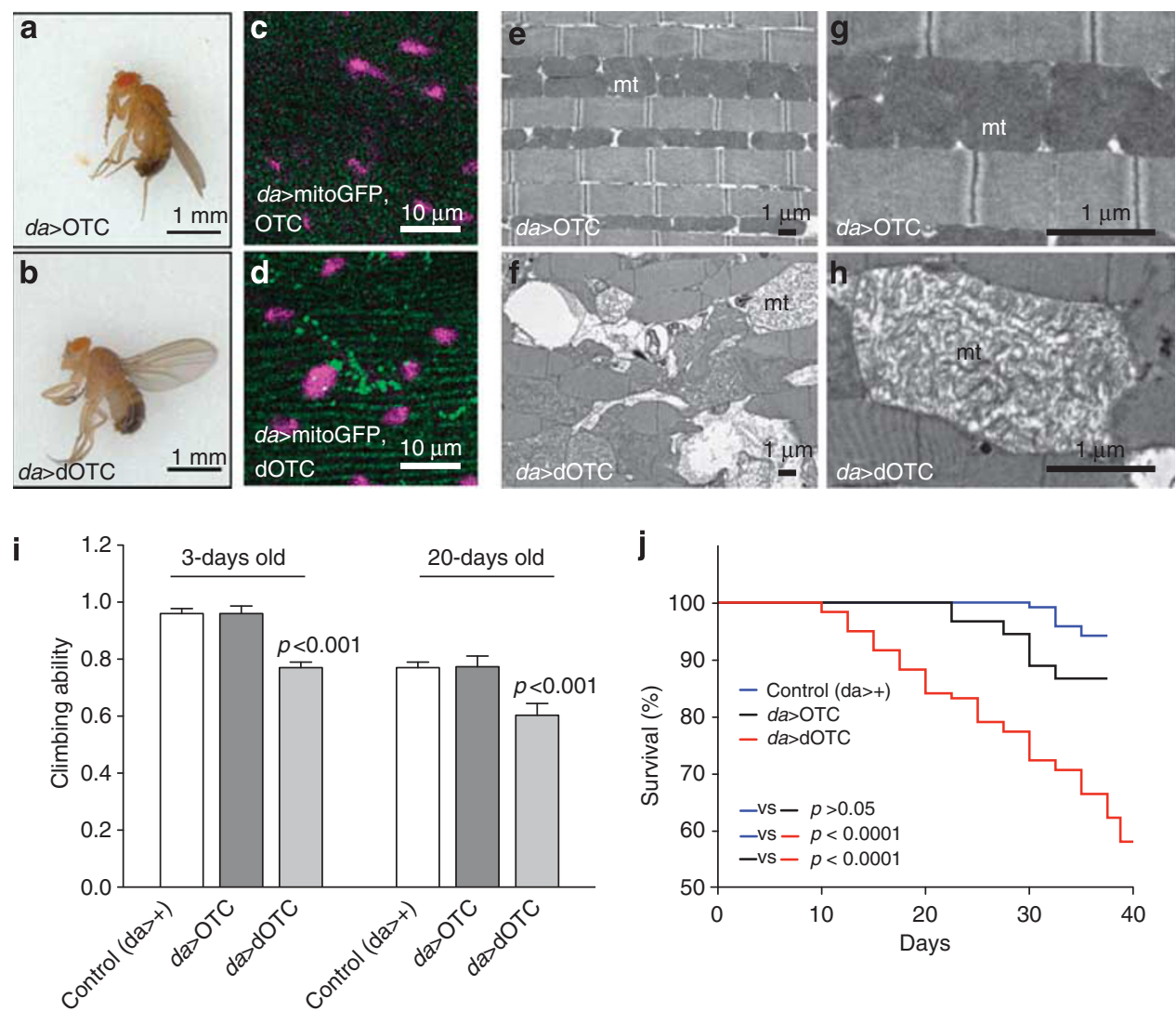

Figure 3 Accumulation of misfolded proteins in mitochondrial phenocopies pink1 and parkin mutant flies. (a and $\mathbf{b})$ Mitochondrial protein misfolding resulted in abnormal wing posture. (c and d) dOTC expression led to the formation of large mitochondrial clusters. Mitochondria were visualised by confocal analysis of the indirect flight muscles in mitochondria-targeted protein (mitoGFP) transgenic animals expressing either OTC or dOTC with da-GAL4 driver. Purple, nuclei; green, mitoGFP. (e-h) Ultrastructural defects in the indirect flight muscles of flies ubiquitously expressing dOTC. Tissues were analysed by transmission electron microscopy (TEM). mt, mitochondria. (i) Mitochondria-targeted protein misfolding caused motor impairment. Control, OTC and dOTC transgenic flies with 3-and 20-days-old were tested using a standard climbing assay (mean \pm S.D., $n \geq 100$ flies per genotype). Statistically significant values relative to control are indicated (two-way ANOVA with Bonferroni post test). (j) dOTC expression resulted in a reduction in total lifespan. Fly viability was scored over a period of 30 days, using a minimum of 200 flies per genotype. The viability of control, OTC and dOTC transgenic flies was compared. Statistical significance is indicated (log-rank, Mantel-Cox test)

the idea that mutations in these PD-related genes in Drosophila might result in a detrimental UPR ${ }^{\mathrm{mt}}$ activation.

Mitochondrial dysfunction induced by protein conformational stress leads to mitochondrial fragmentation and the induction of autophagy. Recently, fission has been shown to mediate the clearance of damaged mitochondria by autophagy, ${ }^{15}$ a recycling programme for impaired and defective cellular components. We confirmed that OTC and dOTC were correctly targeted to mitochondria, by co-expressing each protein with a mitochondrial-targeted GFP reporter in larval tissues using the en-GAL4 driver. Interestingly, we noted a significant degree of fragmentation of the mitochondrial network in the larval wing disc expressing dOTC (Figures 4a-c), suggesting an imbalance towards fission, and therefore we decided to test whether autophagy was being activated in these transgenic animals. In order to study autophagy, we made use of a germline driver nosGAL4 to express GFP-LC3 in larvae. By western blot analysis in adult flies, we showed that dOTC-induced mitochondrial dysfunction was accompanied by accumulation of the autophagy marker LC3-II (Figure 4d). The activation of the autophagic pathway was further confirmed by confocal (Figures 4e-g) and ultrastructural (Supplementary Figures S3A-D) analysis of the larval fat body, a tissue commonly used to study mechanisms of autophagy in flies. We recently proposed a model (reviewed in de Castro et al. $^{2}$ ) where mitochondrial damage resulting in ATP loss could possibly lead to the activation of the energy-sensing kinase AMPK. Flies expressing dOTC show low levels of ATP and increased levels of AMPK phosphorylation (Figure 4h). To test whether AMPK is a key mediator of dOTC-induced autophagic activation, we downregulated the expression of this kinase using RNA interference (Figure 4i). Upon partial loss of AMPK and of its kinase activity, dOTC expression failed to trigger the accumulation of the autophagic marker, LC3-II (Figure 4j). This suggests that AMPK can have a role in the activation of autophagy as a protective mechanism that ensures both the removal of defective mitochondria as well as some recycling of macromolecules required for anabolic processes.

Parkin-mediated enhancement of autophagy suppressed mitochondrial dysfunction induced by protein conformational stress. Recently, it has been proposed that Pink1 and Parkin might be involved in a common pathway 
a

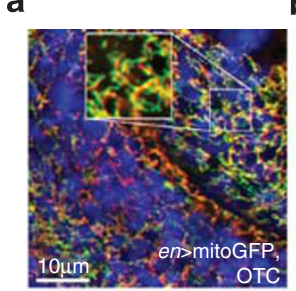

b

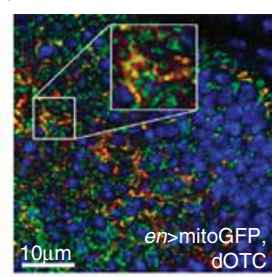

e
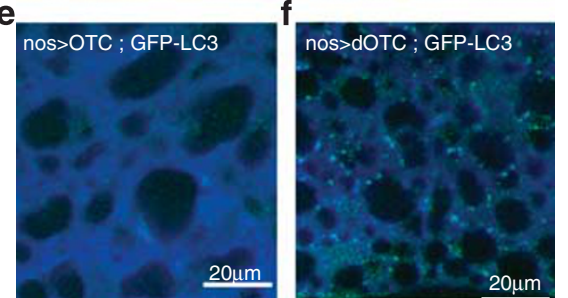

C

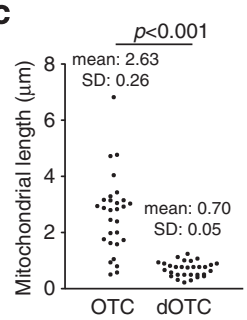

\section{g}

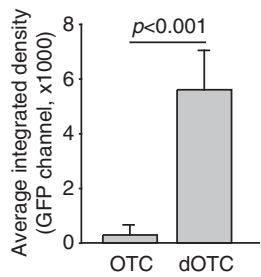

d

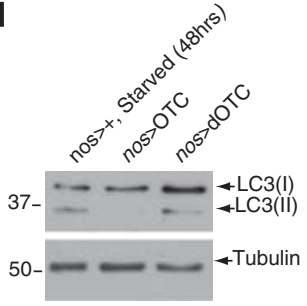

h

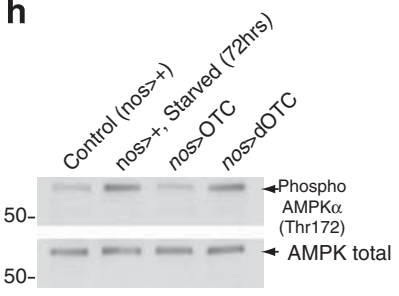

i

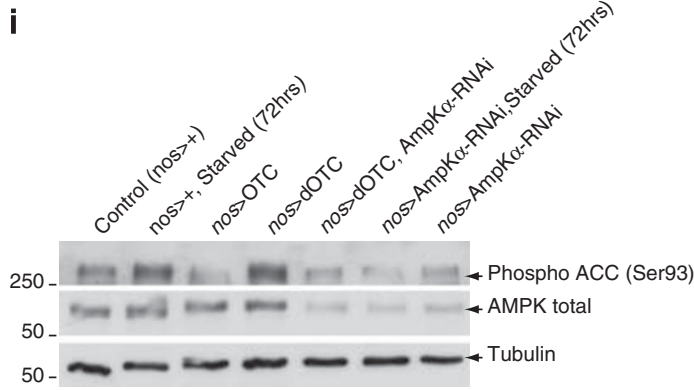

j

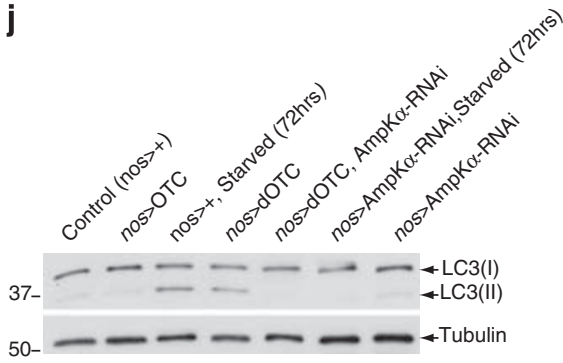

Figure 4 Mitochondrial dysfunction following protein conformational stress results in mitochondrial fragmentation, AMPK phosphorylation and induction of autophagy. (a and $\mathbf{b}$ ) dOTC expression resulted in mitochondrial fragmentation. Confocal analysis of the posterior compartment of larval wing discs co-expressing OTC or dOTC and a mitochondria-targeted protein (mitoGFP) under the control of the en-GAL4 driver. Red, OTC or dOTC; blue, nuclei; green, mitoGFP. (c) Quantification of mitochondrial length in larval wing discs expressing OTC or dOTC. Mean \pm S.D. are shown. Statistically significant values relative to OTC are indicated (unpaired $t$-test). (d) dOTC expressioninduced autophagy. Lysates prepared from adult flies expressing OTC and dOTC under the control of the nos-GAL4 driver were subjected to western blot analysis with the indicated antibodies. Non-lipidated LC3 (GFP-LC3-I, $43 \mathrm{kDa}$ ) and lipidated LC3 (GFP-LC3-II, $36 \mathrm{kDa}$ ) are indicated with arrows. Adult flies were starved for $48 \mathrm{~h}$ as a positive control for the activation of autophagy. (e and $\mathbf{f}$ ) Autophagy was induced upon accumulation of misfolded proteins in the mitochondria. Autophagy was detected in the larval fat body of transgenic animals by confocal microscopy. Induction of dOTC expression led to a redistribution of GFP-LC3 from a uniform to a punctate pattern. (g) Quantification of the GFP-LC3 signal intensity (mean \pm S.D.; $n=3$ ). Statistically significant values relative to OTC are indicated (unpaired $t$-test). (h) dOTC expression-induced AMPK phosphorylation. Lysates prepared from adult flies were subjected to western blot analysis with the indicated antibodies. As a control for the activation of AMPK, control flies were starved for $72 \mathrm{~h}$. (i) RNAi-mediated suppression of AMPK. Downregulation of Ampk was confirmed by measuring the protein levels of total AMPK in the indicated genotypes. Phosphorylation of the AMPK substrate, acetyl-CoA carboxylase (ACC) was used as a marker for AMPK kinase activity. Lysates prepared from adult flies were subjected to western blot analysis with the indicated antibodies. Adult flies were starved for $72 \mathrm{~h}$ as a positive control for AMPK activation and consequent ACC phosphorylation. (j) AMPK acts as an autophagic-triggering sensor following mitochondrial stress. RNAi-mediated knockdown of Ampk blocked the lipidation of LC3 upon dOTC expression or starvation. Lysates prepared from adult flies were subjected to western blot analysis with the indicated antibodies. Non-lipidated LC3 (GFP-LC3-I, 43 kDa) and lipidated LC3 (GFP-LC3-II, $36 \mathrm{kDa}$ ) are indicated with arrows. Adult flies were starved for $72 \mathrm{~h}$ as a positive control for the activation of autophagy

controlling mitochondrial morphology, function and degradation. Several groups have reported that loss of pink1 or parkin in Drosophila leads to very similar mitochondrial phenotypes, and although pink1 is unable to rescue the defects seen in parkin mutants, overexpression of parkin rescues the mitochondrial pathology seen in pink 1 mutants, indicating that pink1 acts genetically upstream of parkin. ${ }^{11,12}$ In mammalian cells, following recruitment by PINK1, Parkin can translocate to damaged mitochondria upon loss of the mitochondrial membrane potential $(\Delta \psi \mathrm{m})$ and act to mediate their removal via a mechanism involving autophagy. 5,6

Thus, we decided to determine the effects of enhanced parkin expression in flies expressing dOTC. We first observed that the climbing defects and abnormal mitochondrial morphology observed in these flies were markedly suppressed by parkin expression (Figure 5a and Supplementary Figures $\mathrm{S} 4 \mathrm{~A}-\mathrm{C}$, respectively). Ultrastructural analysis of the indirect flight muscles also revealed a marked recovery of tissue structure and mitochondrial integrity (Figures $5 d$ and e), and moreover, respiration defects and ATP levels were partially rescued by parkin expression (Supplementary Figures S4D-F), suggesting that Parkin can compensate for protein conformational stress in mitochondria. We proceeded to analyse autophagy by comparing the levels of LC3-II in these transgenic flies. Parkin expression was sufficient to induce autophagy to a degree similar to that observed in control flies in which autophagy was induced by starvation (Figure $5 b$ ). Interestingly, co-expression of dOTC and Parkin led to an increased accumulation of LC3-II, suggesting that Parkin can enhance autophagy upon mitochondrial stress as a protective 


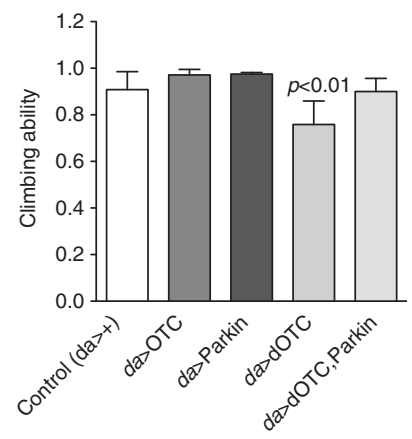

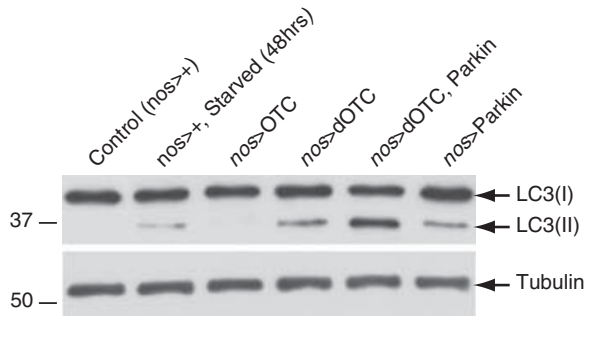

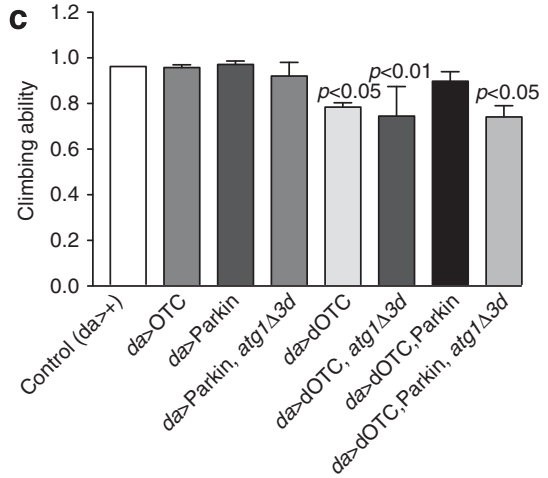

d

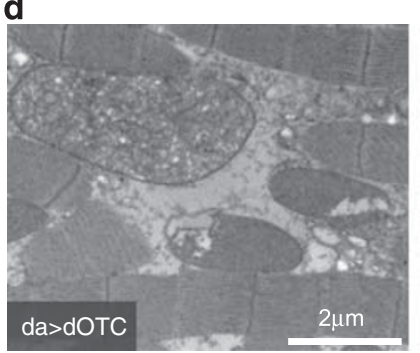

e

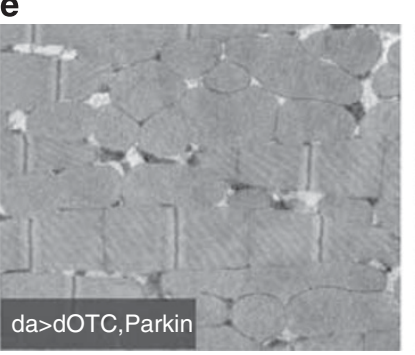

f

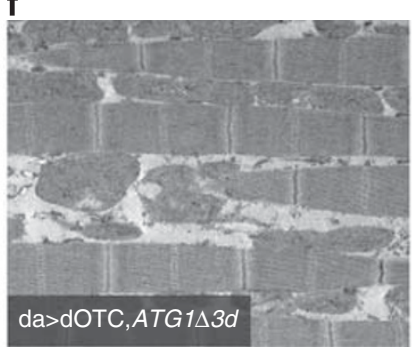

g

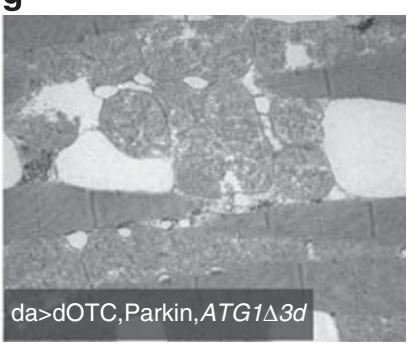

Figure 5 Mitochondrial dysfunction following protein conformational stress is suppressed by Parkin-mediated enhancement of autophagy. (a) Parkin expression rescued the climbing defects caused by the expression of unfolded proteins in mitochondria. The 3-day-old flies with the indicated genotypes were tested using a standard climbing assay (mean \pm S.D., $n \geq 60$ flies for each genotype). Statistically significant values relative to control are indicated (one-way ANOVA with Dunnett's multiple comparison test). (b) Parkin enhanced autophagy in flies expressing dOTC. Whole-fly lysates were subjected to western blotting with the indicated antibodies. Non-lipidated LC3 (GFP-LC3-I, $43 \mathrm{kDa}$ ) and lipidated LC3 (GFP-LC3-II, $36 \mathrm{kDa}$ ) are indicated with arrows. Adult flies were starved for $48 \mathrm{~h}$ as a positive control for the activation of autophagy. (c) Autophagy was required for Parkin-dependent suppression of the dOTC-induced motor impairment. Flies were tested using a standard climbing assay (mean \pm S.D., $n \geq 60$ flies for each genotype). Statistically significant values relative to control are indicated (one-way ANOVA with Dunnett's multiple comparison test). (d-g) Suppression of the indirect flight muscle defects of dOTC-expressing flies by Parkin is autophagy dependent. Ultrastructural analysis of the indirect flight muscles shows that Parkin expression is no longer able to suppress the muscle disorganisation caused by the expression of dOTC in atg1 mutant background

mechanism (Figure 5b). To confirm the requirement of autophagy in this Parkin-mediated protective effect, we tested the epistatic effects of a mutation in the autophagy-regulating kinase, atg1. Parkin suppression of the dOTC-induced climbing defects, tissue structure and mitochondrial integrity were absent in atg1 mutants (Figures 5c, f and g), suggesting that autophagy is required for Parkin-mediated rescue of flies expressing dOTC.

Drosophila ref(2)P is required for parkin-mediated suppression of mitochondrial dysfunction induced by protein conformational stress. Parkin encodes a multifunctional E3 ubiquitin ligase that mediates its own ubiquitination and that of other target proteins. ${ }^{16}$ Mitochondrial translocation of Parkin involves the formation of polyubiquitin chains on specific mitochondrial proteins such as VDAC1 and Mitofusin, and this process seems to be involved in Parkin-dependent mitophagy. ${ }^{17,18}$

The mammalian protein p62/SQSTM1 is an autophagy receptor that can interact with ubiquitin conjugated to a target and LC3/GABARAP on the autophagosome (reviewed in Kirkin et $a l^{19}$ ). It has recently been reported that p62/ SQSTM1 is critical for PINK1/Parkin-mediated mitophagy. ${ }^{17}$ Thus, we determined whether p62/SQSTM1 could act as an adaptor protein in the final step of the mitochondrial autophagy. Co-immunoprecipitation experiments in mammalian cells revealed a strong interaction between p62 and Parkin
(Figure 6a). Interestingly, removal of the ubiquitin-like domain (UBL) from Parkin had no effect on the formation of Parkinp62 complexes.

As Parkin and p62 seemed to physically interact, we next tested whether the single Drosophila p62 orthologue, ref(2) $P$, CG10360, could act genetically downstream of parkin to promote the autophagic clearance of damaged mitochondria. Ref(2)P contains three domains involved in protein-protein interaction: a PB1 domain involved in multimerisation, a $\mathrm{ZZ}$ zinc finger and a ubiquitin-associated domain (UBA). Drosophila ref(2) $P^{o d 2}$ mutants lack the PB1 domain whereas ref(2) $P^{o d 3}$ mutants lack the UBA domain. ${ }^{20,21}$ Both mutants are viable but completely male sterile. ${ }^{21}$ Interestingly, dysfunction in both the Pink1 and Parkin pathways is linked to male sterility because of defective mitochondria. ${ }^{12,13}$ Analysis of the indirect flight muscles in $\operatorname{ref}(2) P^{o d 2}$ and ref(2) $P^{o d 3}$ mutants revealed vacuolisation of mitochondrial cristae in both mutants (Figure 6b), suggesting that defects in ref(2)P are linked to mitochondrial dysfunction.

To determine the role of ref(2)P in the clearance of damaged mitochondria, we tested its requirement in parkin-mediated suppression of the phenotypes observed in dOTC-expressing flies. Parkin was unable to suppress the climbing deficits observed in dOTC flies carrying ref(2)P mutations (Figure 6c). Additionally, Parkin also failed to suppress the mitochondrial loss, addressed through the analysis of the levels of mitochondrial complex I, caused by dOTC in ref(2)P mutants 

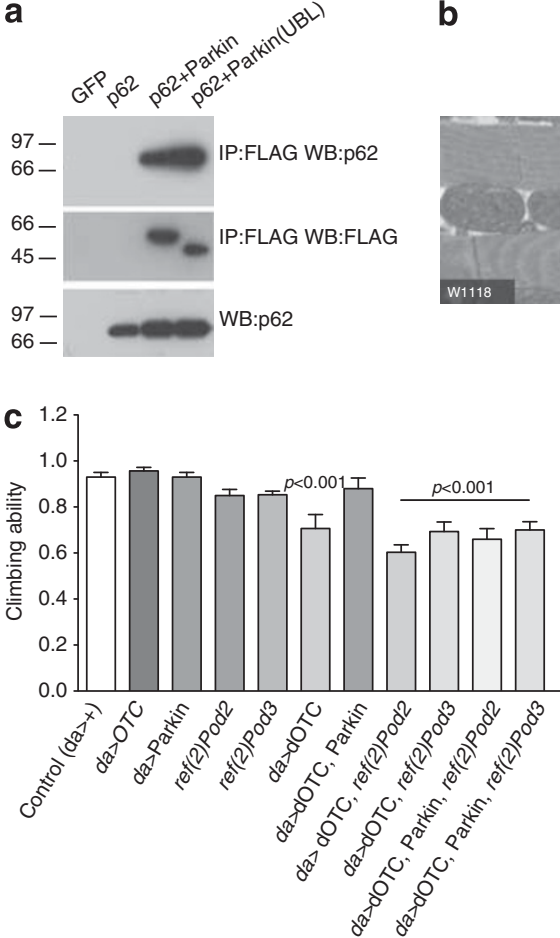

b

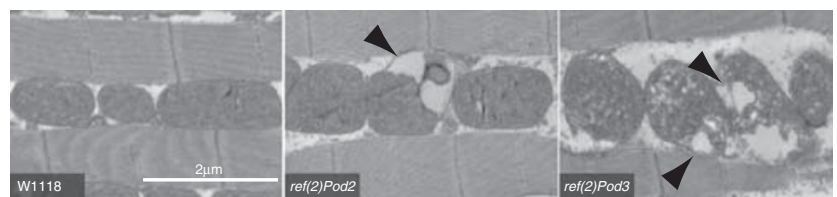

d

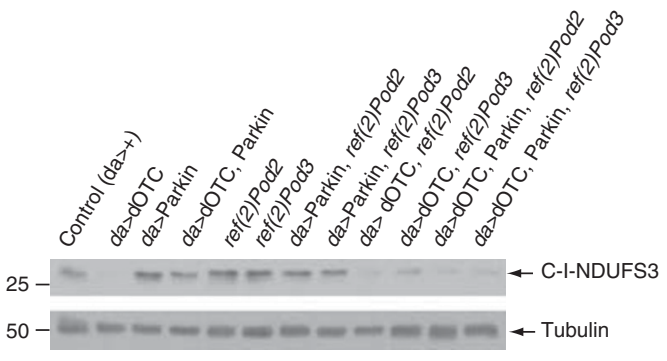

Figure 6 Drosophila ref(2)P is required for parkin-mediated suppression of the phenotype caused by protein conformational stress in mitochondria. (a) Immunoprecipitation of Parkin/p62 complexes in cultured cells. Human HEK-293 cells were transfected with the indicated constructs and incubated for $16 \mathrm{~h}$. Lysates were subjected to immunoprecipitation using an anti-FLAG antibody and analysed by western blotting as indicated. (b) ref(2) $P^{O D 2}$ and ref(2)P ${ }^{O D 3}$ mutants exhibit mitochondrial defects in the indirect flight muscles. Ultrastructural defects in the thoracic muscles were analysed by TEM. Arrowheads point to mitochondrial vacuoles in the mutants. (c) ref(2)P was required for the Parkin-mediated suppression of the motor impairment in dOTC-expressing flies. The 3-day-old flies with the indicated genotypes were tested using a standard climbing assay (mean \pm S.D., $n \geq 60$ flies for each genotype). Statistically significant values relative to control are indicated (one-way ANOVA with Dunnett's multiple comparison test). (d) ref(2)P is required for Parkin-mediated suppression of mitochondrial protein loss in dOTC-expressing flies. The mitochondrial protein loss was addressed by measuring the levels of the nuclear-encoded subunit of mitochondrial complex I (NDUFS3). Flies were analysed by western blotting using the indicated antibodies

(Figure 6d). This suggests that $\operatorname{ref}(2) P$ works genetically downstream of parkin to promote the clearance of mitochondria carrying enhanced levels of misfolded proteins.

\section{Discussion}

Late onset neurodegenerative diseases like PD are associated with the formation of toxic protein aggregates. It has been proposed that in amyotrophic lateral sclerosis (ALS), the toxicity of ALS-linked SOD1 mutations results from the selective accumulation and aggregation of SOD1 mutant protein in spinal cord mitochondria, ${ }^{22,23}$ indicating that mitochondrial protein misfolding may contribute to neurodegeneration in humans. Here, we have performed an in vivo characterisation of a mitochondrial stress-responsive pathway likely to respond to protein misfolding in this organelle. We have determined that defects in Pink1 and Parkin are associated with protein misfolding of components of the respiratory complexes in mitochondria. By generating and characterising an in vivo model of mitochondrial protein misfolding, we obtained evidence that this defect is closely linked to the phenotypic changes observed in the PD-related Drosophila mutants, pink1 and parkin. Importantly, our data suggests that ref(2)P has a critical role in mediating autophagy of defective mitochondria, suggesting that this protein might be a critical effector in an organellar quality control pathway that acts when molecular quality control mechanisms in mitochondria are overwhelmed by excessive protein misfolding. PD is characterised by a selective loss of dopaminergic neurons in the substantia nigra. We targeted the expression of dOTC to dopaminergic neurons and failed to detect any loss of dopaminergic neurons in dOTC-expressing flies (Supplementary Figure S1F). It is noteworthy that there are conflicting reports concerning the role of Drosophila pink1 in dopaminergic neurodegeneration. ${ }^{11,12}$ It is therefore difficult to conclude whether dopaminergic neurons in flies show enhanced sensitivity to mitochondrial dysfunction as a consequence of protein misfolding.

Comparatively, little is known about the contribution of protein aggregation in the mitochondria to age-related neurodegenerative diseases such as PD. Interestingly, it has recently been shown that in the multicellular organism Caenorhabditis elegans, widespread protein insolubility and aggregation are inherent parts of aging and are likely to influence total lifespan and the onset of neurodegenerative diseases..$^{24}$ Our data is compatible with a model where UPR ${ }^{\mathrm{mt}}$ activation might be an early marker of mitochondrial dysfunction in neurodegenerative diseases. As mitochondrial dysfunction was reported to be linked to other neurodegenerative disorders such as Alzheimer's disease and Huntington's disease (reviewed in de Castro et al. ${ }^{2}$ ), it would be important to determine whether the activation of the UPR ${ }^{\mathrm{mt}}$ is also 
associated with these conditions. Nevertheless, given the technical challenges with obtaining robust data on transcriptional changes derived from the analysis of post-mortem human brains, it is likely that model organisms like Drosophila where these diseases can be initially modelled, could provide important clues regarding the link between UPR ${ }^{\mathrm{mt}}$ activation and neurodegenerative diseases in general.

AMPK is a major sensor of the cellular AMP:ATP ratio and is activated by metabolic stresses that inhibit ATP production (reviewed in Hardie ${ }^{25}$ ). We observed an enhanced phosphorylation of both the energy-sensing kinase AMPK and its downstream target acetyl-CoA carboxylase (ACC) upon dOTC expression in flies. It is tempting to propose that, upon mitochondrial dysfunction caused by protein misfolding, AMPK senses a loss of ATP and has a protective role by activating autophagy and ensuring both the removal of defective mitochondria as well as some recycling of macromolecules required for anabolic processes. It is therefore plausible that enhancing AMPK activity might have a positive role to promote efficient mitochondrial quality control.

It is interesting that parkin expression led to an almost complete recovery of mitochondrial integrity in flies expressing dOTC (Supplementary Figures S4A-E). We reason that besides clearing defective mitochondria through autophagy, Parkin can possibly promote the replacement of defective mitochondria with viable organelles. Mammalian PARKIN has been recently shown to indirectly regulate the function of PGC-1 $1 \alpha$, a transcriptional co-activator of genes involved in mitochondrial biogenesis. ${ }^{26}$ This regulation involves the ubiquitination of the PGC- $1 \alpha$ transcriptional repressor PARIS (ZNF746). It is attractive to propose that in Drosophila, the recovery of viable mitochondria promoted by Parkin involves not only the efficient clearance of defective organelles through autophagy but also an enhancement of PGC- $1 \alpha$ activity through the regulation of a putative Drosophila orthologue of PARIS.

Our data suggest that a pathway involving pink1, parkin and ref(2) $P$ has an important role in the maintenance of a viable pool of mitochondria, possibly by ensuring that mitochondria containing excessive levels of unfolded proteins are selectively recycled via autophagy. In humans, mitochondria are the major suppliers of energy to the brain. Neurons have low glycolytic rates, ${ }^{27}$ and therefore rely heavily on mitochondria for energy production. Mitochondria are also capable of buffering cytoplasmic calcium, and thus have a role in the regulation of intracellular calcium dynamics. It is therefore possible that defects in mitochondrial quality control have major implications for both neuronal survival and efficient synaptic transmission.

Although highly speculative, it is tempting to suggest that enhancing pathways that promote autophagy in humans might delay age-related diseases by promoting a healthy pool of viable mitochondria in neuronal cells, sustaining energy demands and efficiently buffering intracellular calcium levels.

\section{Materials and Methods}

A detailed description of all methods used in this study can be found in the Supplementary Information.
Antibodies. The primary antibodies used in this study are listed in Supplementary Table SI.

Genetics and Drosophila strains. Transgenic strains with UAS-OTC and UAS-dOTC were generated by P-element-mediated transformation. Transgenic flies were generated at the CRUK Fly Facility. At least eight independent transgenic lines were derived for each transgene, and all were tested for protein expression. The GAL4 driver lines used were en-GAL4, gmr-GAL4 and da-GAL4. The reporter strains for ER stress and UPS were UAS-xbp1-EGFP ${ }^{28}$ and UAS-G76V-GFP, ${ }^{29}$ respectively. The fly UAS GFP-LC3 lines were obtained from the Bloomington Stock Center (Indiana University, Bloomington, IN, USA). The pink1 $1^{B 9}$, UAS-park ${ }^{c 2}$ and park ${ }^{25}$ flies were a gift from A. Whitworth (MRC, Centre for Developmental and Biomedical Genetics, University of Sheffield, Sheffield, UK). The atg $1^{13 d}$ line ${ }^{30}$ was kindly provided by Dr. TP Neufeld (University of Minnesota, Minneapolis, MN, USA). The ref(2)Pod2 and ref(2) $P^{o d 3}$ lines ${ }^{20}$ were described by Wyers et al. ${ }^{21}$ and were a kind gift from Dr. I Nezis (Institute for Cancer Research, Oslo, Norway). The AmpK $\alpha$-RNAi line (transformant ID 106200) was obtained from Vienna Drosophila RNAi Center (Vienna, Austria). Genotypes for all the flies used in this study are listed in Supplementary Table SII.

Immunofluorescence and confocal microscopy. To analyse the mitochondria in wing discs, third instar larvae were dissected in PBS, fixed in 4\% paraformaldehyde, incubated with Hoechst 33342, stained with anti-Flag antibodies and imaged on a Zeiss (Jena, Germany) LSM510 confocal microscope. For fat body analysis, fed larvae at the appropriate stage were dissected in PBS, fixed in $4 \%$ paraformaldehyde and incubated with Hoechst 33342. Fat bodies were transferred to Vectashield (Vector Laboratories, Burlingame, CA, USA) mounting medium on glass slides, covered and immediately imaged on a Zeiss LSM510 confocal microscope. Adult fly thoraces were dissected, fixed in $4 \%$ paraformaldehyde and incubated with Hoechst 33342 . The indirect flight muscle fibres were isolated and imaged on a Zeiss LSM510 confocal microscope.

Statistical analysis. Data are presented as mean values, and error bars indicate \pm S.D. or \pm S.E.M., as noted. Inferential statistical analysis was performed using the Prism and StatMate software packages (http://www.graphpad.com).

\section{Conflict of Interest}

The authors declare no conflict of interest.

Acknowledgements. We thank Nic Tapon, Pedro Domingos, Udai B Pandey, Alex Whitworth, Thomas Neufeld, loannis Nezis and the Bloomington Drosophila Stock Center for fly stocks; Terence Gilbank and Steve Murray for embryo injections; Judy McWilliam and Tim Smith for assistance with Electron Microscopy; Helen C Ardley for human Parkin expression constructs; Nick Harper for a p62 expression construct and Jalpa Parmar for fly food preparation. Inês Castro is recipient of a Ph.D. Grant (SFRH/BD/41682/2007) from FCT Portugal.

1. Rubinsztein DC. The roles of intracellular protein-degradation pathways in neurodegeneration. Nature 2006; 443: 780-786.

2. de Castro IP, Martins LM, Loh SH. Mitochondrial quality control and Parkinson's disease: a pathway unfolds. Mol Neurobiol 2011; 43: 80-86.

3. Plun-Favreau H, Klupsch K, Moisoi N, Gandhi S, Kjaer S, Frith D et al. The mitochondrial protease HtrA2 is regulated by Parkinson's disease-associated kinase PINK1. Nat Cell Biol 2007; 9: 1243-1252.

4. Moisoi N, Klupsch K, Fedele V, East P, Sharma S, Renton A et al. Mitochondrial dysfunction triggered by loss of HtrA2 results in the activation of a brain-specific transcriptional stress response. Cell Death Differ 2009; 16: 449-464.

5. Narendra D, Tanaka A, Suen DF, Youle RJ. Parkin is recruited selectively to impaired mitochondria and promotes their autophagy. J Cell Biol 2008; 183: 795-803.

6. Narendra DP, Jin SM, Tanaka A, Suen DF, Gautier CA, Shen J et al. PINK1 is selectively stabilized on impaired mitochondria to activate Parkin. PLOS Biol 2010; 8: e1000298.

7. Broadley SA, Hartl FU. Mitochondrial stress signaling: a pathway unfolds. Trends Cell Biol 2008; 18: 1-4.

8. Zhao Q, Wang J, Levichkin IV, Stasinopoulos S, Ryan MT, Hoogenraad NJ. A mitochondrial specific stress response in mammalian cells. Embo J 2002; 21: 4411-4419.

9. Brand $\mathrm{AH}$, Perrimon $\mathrm{N}$. Targeted gene expression as a means of altering cell fates and generating dominant phenotypes. Development 1993; 118: 401-415.

10. Haynes CM, Petrova K, Benedetti C, Yang $Y$, Ron D. ClpP mediates activation of a mitochondrial unfolded protein response in C.elegans. Dev Cell 2007; 13: 467-480. 
11. Park J, Lee SB, Lee S, Kim Y, Song S, Kim S et al. Mitochondrial dysfunction in Drosophila PINK1 mutants is complemented by parkin. Nature 2006; 441: 1157-1161.

12. Clark IE, Dodson MW, Jiang $\mathrm{C}, \mathrm{Cao} \mathrm{JH}$, Huh JR, Seol JH et al. Drosophila pink1 is required for mitochondrial function and interacts genetically with parkin. Nature 2006; 441: 1162-1166.

13. Greene JC, Whitworth AJ, Kuo I, Andrews LA, Feany MB, Pallanck LJ. Mitochondrial pathology and apoptotic muscle degeneration in Drosophila parkin mutants. Proc Natl Acad Sci USA 2003; 100: 4078-4083.

14. Pesah $Y$, Pham T, Burgess H, Middlebrooks B, Verstreken $P$, Zhou $Y$ et al. Drosophila parkin mutants have decreased mass and cell size and increased sensitivity to oxygen radical stress. Development 2004; 131: 2183-2194.

15. Twig G, Elorza A, Molina AJ, Mohamed H, Wikstrom JD, Walzer G et al. Fission and selective fusion govern mitochondrial segregation and elimination by autophagy. EMBO J 2008; 27: 433-446.

16. Kahle PJ, Haass C. How does parkin ligate ubiquitin to Parkinson's disease? EMBO Rep 2004; 5: 681-685.

17. Geisler S, Holmstrom KM, Skujat D, Fiesel FC, Rothfuss OC, Kahle PJ et al. PINK1/Parkinmediated mitophagy is dependent on VDAC1 and p62/SQSTM1. Nat Cell Biol 2010; 12: 119-131.

18. Ziviani E, Tao RN, Whitworth AJ. Drosophila parkin requires PINK1 for mitochondrial translocation and ubiquitinates mitofusin. Proc Natl Acad Sci USA 2010; 107: 5018-5023.

19. Kirkin V, McEwan DG, Novak I, Dikic I. A role for ubiquitin in selective autophagy. Mol Cell 2009; 34: 259-269.

20. Nezis IP, Simonsen A, Sagona AP, Finley K, Gaumer S, Contamine D et al. Ref(2)P, the Drosophila melanogaster homologue of mammalian p62, is required for the formation of protein aggregates in adult brain. J Cell Biol 2008; 180: 1065-1071.

21. Wyers F, Petitjean AM, Dru P, Gay P, Contamine D. Localization of domains within the Drosophila Ref(2)P protein involved in the intracellular control of sigma rhabdovirus multiplication. J Virol 1995; 69: 4463-4470.
22. Liu J, Lillo C, Jonsson PA, Vande Velde C, Ward CM, Miller TM et al. Toxicity of familial ALS-linked SOD1 mutants from selective recruitment to spinal mitochondria. Neuron 2004; 43: 5-17.

23. Pasinelli $\mathrm{P}$, Belford ME, Lennon N, Bacskai BJ, Hyman BT, Trotti D et al. Amyotrophic lateral sclerosis-associated SOD1 mutant proteins bind and aggregate with $\mathrm{Bcl}-2$ in spinal cord mitochondria. Neuron 2004; 43: 19-30.

24. David DC, Ollikainen N, Trinidad JC, Cary MP, Burlingame AL, Kenyon C. Widespread protein aggregation as an inherent part of aging in C.elegans. PLOS Biol 2010; 8 e1000450.

25. Hardie DG. AMP-activated/SNF1 protein kinases: conserved guardians of cellular energy. Nat Rev Mol Cell Biol 2007; 8: 774-785.

26. Shin JH, Ko HS, Kang H, Lee Y, Lee YI, Pletinkova $O$ et al. PARIS (ZNF746) repression of PGC-1alpha contributes to neurodegeneration in Parkinson's disease. Cell 2011; 144 : 689-702.

27. Bolanos JP, Almeida A. The pentose-phosphate pathway in neuronal survival against nitrosative stress. IUBMB Life 2010; 62: 14-18.

28. Ryoo HD, Domingos PM, Kang MJ, Steller H. Unfolded protein response in a Drosophila model for retinal degeneration. EMBO J 2007; 26: 242-252.

29. Pandey UB, Nie Z, Batlevi Y, McCray BA, Ritson GP, Nedelsky NB et al. HDAC6 rescues neurodegeneration and provides an essential link between autophagy and the UPS. Nature 2007; 447: 859-863.

30. Scott RC, Schuldiner O, Neufeld TP. Role and regulation of starvation-induced autophagy in the Drosophila fat body. Dev Cell 2004; 7: 167-178.

(c) This work is licensed under the Creative Commons

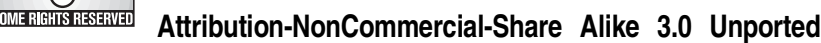
License. To view a copy of this license, visit http://creativecommons. org/licenses/by-nc-sa/3.0/

Supplementary Information accompanies the paper on Cell Death and Differentiation website (http://www.nature.com/cdd) 